\title{
A NEW CHARACTERIZATION OF BESICOVITCH ALMOST PERIODIC FUNCTIONS
}

\author{
ABDALLAH N. DABBOUCY and HENRY W. DAVIS*
}

\section{Introduction and history.}

The set of Besicovitch almost periodic functions, $\left\{\mathrm{B}^{p}-\mathrm{AP}\right\}$, may be defined as the closure of the Bohr almost periodic functions via the Besicovitch norm $\|\cdot\|_{B(p)}$, where

$$
\|f\|_{B(p)}=\varlimsup_{T \rightarrow \infty}\left[\frac{1}{2 T} \int_{-T}^{T}|f(x)|^{p} d x\right]^{1 / p},
$$

and $p \in[1, \infty)$ is a fixed parameter. Several authors have given structural characterizations which assure that a function, known to be in $L_{p}(-T, T)$ for all $T>0$, is also in $\left\{\mathrm{B}^{p}-\mathrm{AP}\right\}$. The first was by Bohr and Besicovitch [2]:

A set $E$ of real numbers is called satisfactorily uniform if there exists $L>0$ such that the ratio of maximum number of elements of $E$ included in an interval of length $L$ to the minimum number is less than 2. Then $f \in\left\{\mathrm{B}^{p}-\mathrm{AP}\right\}$ if and only if for every $\varepsilon>0$ the set of $\|\cdot\|_{B(p)^{-}}$-translation numbers,

$$
B^{p} E(\varepsilon, f)=\left\{u \in R:\left\|f_{u}-f\right\|_{B(p)}<\varepsilon\right\},
$$

contains a satisfactorily uniform subset,

such that

$$
\ldots u_{-2}<u_{-1}<u_{0}<u_{1}<u_{2}<\ldots \text {, }
$$

$$
\bar{M}_{x} \bar{M}_{i}\left[\frac{1}{c} \int_{x}^{x+c}\left|f_{u_{i}}(t)-f(t)\right|^{p} d t\right]<\varepsilon^{p}
$$

whenever $c>0$. Here $f_{u}(x)=f(x+u)$,

$$
\bar{M}_{x}[g(x)]=\varlimsup_{T \rightarrow \infty} \frac{1}{2 T} \int_{-T}^{T} g(x) d x,
$$

Received August 20, 1970; in revised form December 10, 1970.

* Part of this work performed under the auspices of the U.S. Atomic Energy Commission. 
and

$$
\bar{M}_{i}[g(i)]=\varlimsup_{k \rightarrow \infty} \frac{1}{2 k+1} \sum_{i=-k}^{k} g(i) .
$$

In the case $p=1$ Besicovitch [1] has shown: $f \in\{\mathrm{B}-\mathrm{AP}\}$ if and only if for every $\varepsilon>0$ there is a satisfactorily uniform set of numbers

such that

$$
\ldots u_{-2}<u_{-1}<u_{0}=0<u_{1}<u_{2}<\ldots
$$

$$
\bar{M}_{x} \bar{M}_{i}\left[\int_{x}^{x+1}\left|f_{u_{i}}(t)-f(t)\right| d t\right]<\varepsilon .
$$

Incidentally, it is easy to see that a structural characterization of $\{\mathrm{B}-\mathrm{AP}\}$ may be turned into a structural characterization of $\left\{\mathrm{B}^{p}-\mathrm{AP}\right\}$ by adjoining the condition

$$
\lim _{n \rightarrow \infty}\left\|f-f_{n}\right\|_{B(p)}=0
$$

where

$$
\begin{aligned}
f_{n}(x) & =f(x) & & \text { if }|f(x)|<n \\
& =n f(x) /|f(x)| & & \text { otherwise }
\end{aligned}
$$

Alternatively, one may adjoin the condition

$$
\bar{M}\left[|f|^{p} \chi_{E}\right] \rightarrow 0 \quad \text { as } \bar{\mu}(E) \rightarrow 0 .
$$

Here $E$ is a measurable set, $f$ is a measurable function, $\chi_{E}$ is the characteristic function of $E$, and we define $\bar{\mu}(E)=\bar{M}\left[\chi_{E}\right]$.

E. Følner [5] has given the following characterization of $\left\{\mathrm{B}^{p}-\mathrm{AP}\right\}$ :

$f \in\left\{\mathrm{B}^{p}-\mathrm{AP}\right\}$ if and only if $f$ satisfies either (1) or $(1)^{\prime}$ and

(2) for every $\varepsilon>0$ there exists a relatively dense set $T=T(\varepsilon)$ and a set $E=E(\varepsilon)$ such that $\bar{\mu}(E)>1-\varepsilon$ and $|f(x+t)-f(x)|<\varepsilon$ whenever $t \in T$, and $x, x+t \in E$.

(Actually Følner did not require that $f \in L_{p}(-T, T)$ for all $T>0$ but only that $f$ be measurable. His characterization then has the third condition that $\bar{\mu}(\{x:|f(x)|=\infty\})=0$.) Finally, R. Doss [4] has proven that

$f \in\{\mathrm{B}-\mathrm{AP}\}$ if and only if

(a) $\|f\|_{B(1)}<\infty$ and $\lim _{u \rightarrow 0}\left\|f_{u}-f\right\|_{B(1)}=0$;

(b) $f$ is $\|\cdot\|_{B(1)}$-normal, that is, from any sequence $b_{n}$ can be extracted a subsequence $c_{n}$ such that

$$
\lim _{m, n \rightarrow \infty}\left\|f_{c_{n}}-f_{c_{m}}\right\|_{B(1)}=0 ;
$$


(c) for any real $\lambda$,

$$
\text { (c } \lambda) \lim _{L \rightarrow \infty} \bar{M}_{x}\left|\frac{1}{L} \int_{x}^{x+L} f(t) e^{i \lambda t} d t-\frac{1}{L} \int_{0}^{L} f(t) e^{i \lambda t} d t\right|=0 .
$$

The conditions $(\mathrm{c} \lambda)$ form an infinity of independent conditions.

Two other very interesting characterizations of $\left\{\mathrm{B}^{p}-\mathrm{AP}\right\}$ are in the literature, one by R. Doss [3] and one by A. S. Kovanko [10]. These involve certain functions $f^{(a)}$ of period $a$, where $a$ runs through the real numbers. We shall not restate them here.

In this paper we show that $f \in\{\mathrm{B}-\mathrm{AP}\}$ if and only if

(A1) $f$ is $\|\cdot\|_{B(1)}$-normal, and

(B) for all but a countable set of $\varepsilon>0$ it is the case that

$$
\bar{M}_{x} \bar{M}_{w}\left[|f(w+x)-f(x)| \chi_{B E(\varepsilon, f)}(w)\right] \leqq \varepsilon \bar{\mu}(B E(\varepsilon, f)) .
$$

(Al) may be replaced by the equivalent condition

(A2) for every $\varepsilon>0$, the set $B E(\varepsilon, f)$ is relatively dense and open.

The requirement in (A2) that $B E(\varepsilon, f)$ be open may be weakened to require only that $B E(\varepsilon, f)$ be of positive measure or of second category. However some sort of "width" requirement on $B E(\varepsilon, f)$ is necessary. Examples illustrating this and other points are discussed in the last section.

\section{The main theorem.}

We begin with a few notational remarks, additional to those made above. We denote by $R$ the set of real numbers and by $\alpha(R)$ the set of (continuous) Bohr almost periodic functions on $R$ while $\mu$ denotes Lebesgue measure on $\mathrm{R}$. If $f$ is a measurable function on $\mathrm{R},\|f\|_{\infty}$ is its essential supremum and $\|f\|$ is its Besicovitch 1-norm:

$$
\|f\|=\varlimsup_{T \rightarrow \infty} \frac{1}{2 T} \int_{-T}^{T}|f| d \mu .
$$

Also

$$
\begin{aligned}
B E(\varepsilon, f) & =\left\{x \in R:\left\|f_{x}-f\right\|<\varepsilon\right\}, \\
E(\varepsilon, f) & =\left\{x \in R:\left\|f_{x}-f\right\|_{\infty}<\varepsilon\right\} .
\end{aligned}
$$

$L_{1, \text { loc }}(\mathrm{R})$ denotes the set of all complex-valued functions $f$ on $\mathrm{R}$ such that $f \in L_{1}(-T, T)$ for all $T>0$. Notice that $\left\|f_{a}\right\|=\|f\|$ for all $f \in$ $L_{1, \text { loc }}(\mathrm{R})$ and all $a \in R$ even when one side is $\infty$, Indeed 


$$
\begin{aligned}
\left\|f_{a}\right\| & =\varlimsup_{T \rightarrow \infty}\left[\frac{1}{2 T} \int_{-T+a}^{T+a}|f| d \mu\right] \\
& \leqq \varlimsup_{T \rightarrow \infty}\left[\frac{2(T+|a|)}{2 T} \frac{1}{2(T+|a|)} \int_{-(T+|a|)}^{T+|a|}|f| d \mu\right]=\|f\|,
\end{aligned}
$$

which includes the opposite inequality. By a $\delta$-mesh in a metric space is meant a finite set of points of the space such that every point of the space is within $\delta$ of one of the points of the finite set. Finally, we introduce two conditions for a function $f \in L_{1,100}(\mathrm{R})$ :

(A3) for every $\varepsilon>0$, the set $B E(\varepsilon, f)$ is relatively dense and either of positive measure or of second category;

(A4) for every $\varepsilon>0$ there exists a finite set $w_{1}, \ldots, w_{n} \in \mathbf{R}$ such that $\mathrm{R}=\bigcup_{i=1}^{n}\left[w_{i}+B E(\varepsilon, f)\right]$.

2.1. Proposition. For a function $f \in L_{1, l o c}(\mathrm{R})$ the conditions (Al), (A2), (A3), (A4) are equivalent.

Proof. Suppose $f$ satisfies (Al) and take $\varepsilon>0$. As $\left(\left\{f_{a}: a \in \mathrm{R}\right\},\|\cdot\|\right)$ is conditionally compact it is totally bounded so it contains an $\varepsilon$-mesh, say $f_{u_{1}}, \ldots, f_{u_{n}}$. If $u \in \mathrm{R}$, then for some $i$

$$
\left\|f_{u-u_{i}}-f\right\|=\left\|f_{u}-f_{u_{i}}\right\|<\varepsilon,
$$

so $u \in u_{i}+B E(\varepsilon, f)$. Thus $\mathrm{R}=\bigcup_{i=1}^{n}\left[u_{i}+B E(\varepsilon, f)\right]$. $\varepsilon$ being arbitrary, $f$ satisfies (A4).

Suppose $f$ satisfies (A4). For each $\varepsilon>0$, the set $B E(\varepsilon, f)$ is clearly measurable and it follows from (A4) that it is of positive measure and of second category. To see that each $B E(\varepsilon, f)$ is relatively dense take $\varepsilon>0$ and

$$
L>2 \sup \left\{\left|w_{i}\right|: 1 \leqq i \leqq n\right\},
$$

where the $w_{i}$ are as in (A4). We claim that $B E(\varepsilon, f)$ meets every interval of the form $(a, a+L), a \in \mathrm{R}$. Indeed if $a \in \mathrm{R}$, then $a+\frac{1}{2} L=w_{i}+b$ for some $b \in B E(\varepsilon, f)$. Hence

$$
b=a+\frac{1}{2} L-w_{i} \in(a, a+L) .
$$

Consequently $B E(\varepsilon, f)$ is relatively dense, whence (A4) implies (A3).

Assuming $f$ satisfies (A3) we show $f$ satisfies (A2) by showing that each $B E(\varepsilon, f)$ is open. Now

$$
B E(\varepsilon, f) \supset\left\{x-y: x, y \in B E\left(\frac{1}{2} \varepsilon, f\right)\right\} .
$$


It follows from the fact that $B E\left(\frac{1}{2} \varepsilon, f\right)$ is Borel and of positive measure or from the fact that $B E\left(\frac{1}{2} \varepsilon, f\right)$ is of second category that the right side, above, contains a neighborhood of 0 (cf. [7, 61.3], [9, Chap. 6, problem P]). Thus each $B E(\varepsilon, f)$ contains a neighborhood of 0 . Now for $\varepsilon>0$ take $b \in B E(\varepsilon, f)$. Take $\varepsilon_{1}>0$ such that

$$
\left\|f_{b}-f\right\|+\varepsilon_{1}<\varepsilon .
$$

Let $\delta>0$ be such that $(-\delta, \delta) \subset B E\left(\varepsilon_{1}, f\right)$. Then, if $|b-c|<\delta$ we have

$$
\begin{aligned}
\left\|f_{c}-f\right\|=\left\|f_{-c}-f\right\| & =\left\|f_{b-c}-f_{b}\right\| \\
& \leqq\left\|f_{b-c}-f\right\|+\left\|f-f_{b}\right\| \\
& <\varepsilon_{1}+\left\|f_{b}-f\right\|<\varepsilon,
\end{aligned}
$$

whence $c \in B E(\varepsilon, f)$. It follows that $B E(\varepsilon, f)$ is open and, $\varepsilon$ being arbitrary, (A3) implies (A2).

Suppose $f$ satisfies (A2) and take $\varepsilon>0$. As $0 \in B E(\varepsilon, f)$ and $B E(\varepsilon, f)$ is open, there exists $\delta>0$ such that $(-\delta, \delta) \subset B E(\varepsilon, f)$. Take $L>0$ such that every interval of length $L$ meets $B E(\varepsilon, f)$. As

$$
B E(2 \varepsilon, f) \supset B E(\varepsilon, f)+B E(\varepsilon, f),
$$

every interval of length $L$ contains an interval of length $\delta$ all of whose points are in $B E(2 \varepsilon, f)$. Let $n$ be an integer larger than $2 L / \delta$. Then

$$
\bigcup_{i=-n}^{n}\left[\frac{1}{2} i \delta+B E(2 \varepsilon, f)\right]=\mathrm{R} .
$$

Consequently $\left\{f_{\frac{1}{2} i \delta}:-n \leqq i \leqq n\right\}$ is a $2 \varepsilon$-mesh in $\left(\left\{f_{a}: a \in \mathrm{R}\right\},\|\cdot\|\right)$. As $\varepsilon>0$ is arbitrary, $\left(\left\{f_{a}: a \in \mathrm{R}\right\},\|\cdot\|\right)$ is totally bounded and (Al) follows. This proves the proposition.

2.2. Lemma. If $f \in\{\mathrm{B}-\mathrm{AP}\}$, then $f$ satisfies (A3). Hence $f$ also satisfies (A1), (A2) and (A4).

Proof. This is well-known and follows from the fact that if $g \in \alpha(\mathbf{R})$ then

$$
\left\|f_{u}-f\right\| \leqq\left\|f_{u}-g_{u}\right\|+\left\|g_{u}-g\right\|+\|g-f\| \leqq 2\|f-g\|+\left\|g_{u}-g\right\|_{\infty} .
$$

Thus if $\|f-g\|<\frac{1}{3} \varepsilon$ we get that $E\left(\frac{1}{3} \varepsilon, g\right) \subset B E(\varepsilon, f)$. The set $E\left(\frac{1}{3} \varepsilon, g\right)$ is relatively dense and contains a neighborhood of 0 , as $g$ is uniformly continuous. Since $\varepsilon$ is arbitrary, $f$ satisfies (A3).

2.3 Lemma. Let $f \in L_{1, \text { loc }}(\mathrm{R})$ satisfy (A2). For $x \in \mathrm{R}$ define $h(x)=$ $\left\|f_{x}-f\right\|$. Then $h \in \alpha(\mathrm{R})$ and for every $\varepsilon>0$,

$$
E(\varepsilon, h)=B E(\varepsilon, f) .
$$


Proof. By (A2), the set $B(1, f)$ contains a neighborhood of 0 , so $h(x)$ is finite for $x$ near 0 . As

$$
h(n x) \leqq\left\|f_{n x}-f_{(n-1) x}\right\|+\ldots+\left\|f_{x}-f\right\|=n h(x),
$$

it follows that $h(x)$ is finite for all $x \in \mathrm{R}$. Take $\varepsilon>0$. From

$$
\left\|h_{u}-h\right\|_{\infty}=\sup _{x \in \mathrm{R}}\left|\left\|f_{x+u}-f\right\|-\left\|f_{x}-f\right\|\right| \leqq \sup _{x \in \mathrm{R}}\left\|f_{x+u}-f_{x}\right\|=\left\|f_{u}-f\right\|
$$

it follows that $B E(\varepsilon, f) \subset E(\varepsilon, h)$. On the other hand, if $u \in E(\varepsilon, h)$, then

$$
\sup _{x \in \mathbf{R}}\left|\left\|f_{x+u}-f\right\|-\left\|f_{x}-f\right\|\right|<\varepsilon .
$$

Letting $x=0$ gives $\left\|f_{u}-f\right\|<\varepsilon$, so $u \in B E(\varepsilon, f)$. Thus $E(\varepsilon, h) \subset B E(\varepsilon, h)$. As $\varepsilon>0$ is arbitrary, we have $E(\varepsilon, h)=B E(\varepsilon, f)$ for all $\varepsilon>0$. That $h \in \alpha(\mathrm{R})$ now follows from the fact that $f$ satisfies (A2).

2.4 Notation. We let $\bar{R}$ denote the Bohr compactification of $R$ and consider $\mathrm{R}$ as a dense subset of $\overline{\mathrm{R}}$. For $f \in \alpha(\mathrm{R})$ we let $\bar{f}$ denote its continuous extension to $\overline{\mathrm{R}}$. Letting $C(\overline{\mathrm{R}})$ denote the set of continuous complex valued functions on $\overline{\mathrm{R}}$, we get that $f \rightarrow \bar{f}$ is a vector space isomorphism from $\alpha(\mathrm{R})$ onto $C(\overline{\mathrm{R}})$. See, for example, [9, pp. 247-249]. If $A \subset \overline{\mathrm{R}}$, we let $A^{\mathrm{C}}$ denote its closure. If $\bar{h} \in C(\overline{\mathrm{R}})$, we define

$$
E(\varepsilon, \bar{h})=\left\{x \in \overline{\mathrm{R}}:\left\|\bar{h}_{x}-\bar{h}\right\|_{\infty}<\varepsilon\right\},
$$

for each $\varepsilon>0$. Finally, we let $v$ denote Haar measure on $\overline{\mathrm{R}}$.

2.5 Lemma. Let $\bar{h} \in C(\bar{R})$. Then for all but a countable set of $\varepsilon>0$ we have

$$
v(E(\varepsilon, \bar{h}))=v\left(E(\varepsilon, \bar{h})^{\mathrm{C}}\right) .
$$

Proof. Take $\varepsilon>0$ and let $A_{s}=\left\{x \in \overline{\mathrm{R}}:\left\|\bar{h}_{x}-\bar{h}\right\|_{\infty}=\varepsilon\right\}$. As $\bar{h}$ is uniformly continuous, $\left\|\bar{h}_{x}-\bar{h}\right\|_{\infty}$ is a continuous function of $x \in \mathrm{R}$. Thus

$$
A_{8} \supset E(\varepsilon, \bar{h})^{\mathrm{C}} \sim E(\varepsilon, \bar{h}) \text {. }
$$

The sets $\left\{A_{\varepsilon}\right\}_{\varepsilon>0}$ are pairwise disjoint. If $v\left(A_{\varepsilon}\right)>0$ for uncountably many $\varepsilon$, we would have that $\nu(\bar{R})=\infty$, contrary to the compactness of $\bar{R}$. Hence for all but a countable set of $\varepsilon>0$ we have $v\left(A_{\varepsilon}\right)=0$. Further $\nu\left(E(\varepsilon, \bar{h})^{\mathrm{C}}\right)=\nu(E(\varepsilon, \bar{h}))$ for such $\varepsilon$.

2.6 Notation. For $h \in \alpha(\mathbf{R})$ we define

$$
T(h)=\left\{\eta>0: v\left(E(\eta, \bar{h})^{\mathrm{C}}\right)=v(E(\eta, \bar{h}))\right\} .
$$

By 2.5, the set $T(h)$ contains all but a countable set of the positive numbers. 
2.7 Lemma. If $h \in \alpha(\mathrm{R})$ and $\eta \in T(h)$, then $\chi_{E(\eta, h)} \in\{\mathrm{B}-\mathrm{AP}\}$.

Proof. Observe that $E(\eta, h)=E(\eta, \bar{h}) \cap \mathrm{R}$ and $E(\eta, \bar{h})$ is open in $\overline{\mathrm{R}}$. Take $\bar{a}_{n} \in C(\overline{\mathrm{R}})$ such that $\bar{a}_{n} \uparrow \chi_{E(\eta, \bar{h})}$ and $\bar{a}_{n}(x) \geqq 0$ for all $x \in \overline{\mathrm{R}}$. Then

$$
0 \leqq \int_{\mathrm{R}}\left(\chi_{E(\eta, \bar{h})}-\bar{a}_{n}\right) d \nu \rightarrow 0 \quad \text { as } n \rightarrow \infty .
$$

As $\eta \in T(h)$, we may apply Theorem 26.17 of [8] to conclude that

$$
\bar{M}\left|\chi_{E(\eta, h)}-a_{n}\right|=M\left[\chi_{E(\eta, h)}-a_{n}\right] \rightarrow 0 \quad \text { as } n \rightarrow \infty .
$$

The lemma follows.

2.8 THEOREM. If $f \in\{\mathrm{B}-\mathrm{AP}\}$, then $f$ satisfies (B).

Proof. Take $f \in\{\mathrm{B}-\mathrm{AP}\}$ and define $h(x)=\left\|f_{x}-f\right\|$ for all $x \in \mathrm{R}$. By 2.2, 2.3 and 2.5 it suffices to show that for all $\varepsilon$ in $T(h)$,

$$
\bar{M}_{x} \bar{M}_{w}\left[|f(w+x)-f(x)| \chi_{B E(\varepsilon, f)}(w)\right] \leqq \varepsilon \bar{\mu}(B E(\varepsilon, f)) .
$$

Take any $\varepsilon$ in $T(h)$. Take $f_{n} \in \alpha(\mathrm{R})$ (and not identially 0 ) such that $\left\|f-f_{n}\right\| \rightarrow 0$ as $n \rightarrow \infty$. By 2.2, 2.3 and 2.7, $\chi_{B E(\varepsilon, f)} \in\{\mathrm{B}-\mathrm{AP}\}$. Take $b_{n} \in \alpha(\mathrm{R})$ such that

$$
\left\|\chi_{B E(\varepsilon, f)}-b_{n}\right\| \leqq 1 /\left(n\left\|f_{n}\right\|_{\infty}\right), \quad n=1,2, \ldots
$$

We shall show that

$$
\begin{aligned}
\bar{M}_{x} \bar{M}_{w}[\mid f(w+x)- & \left.f(x) \mid \chi_{B E(\varepsilon, f)}(w)\right] \\
& =\lim _{n \rightarrow \infty} M_{x} M_{w}\left[\left|f_{n}(w+x)-f_{n}(x)\right| b_{n}(w)\right] .
\end{aligned}
$$

For any fixed $x \in \mathrm{R}$, we have $|f(w+x)-f(x)| \in\{\mathrm{B}-\mathrm{AP}\}$ whence also

$$
|f(w+x)-f(x)| \chi_{B E(\varepsilon, f)}(w) \in\{\mathrm{B}-\mathrm{AP}\}
$$

(cf. [6, page 7]). Thus $M_{w}\left[|f(w+x)-f(x)| \chi_{B E(\varepsilon, f)}(w)\right]$ exists for each $x \in \mathrm{R}$. Also

$$
\begin{aligned}
& \bar{M}_{x}\left|M_{w}\left[|f(w+x)-f(x)| \chi_{B E(\varepsilon, f)}(w)\right]-M_{w}\left[\left|f_{n}(w+x)-f_{n}(x)\right| b_{n}(w)\right]\right| \\
& \leqq \bar{M}_{x}\left|M\left[\left(\left|f_{x}-f(x)\right|-\left|f_{n x}-f_{n}(x)\right|\right) \chi_{B E(\varepsilon, f)}\right]\right| \\
& \quad \bar{M}_{x} \mid M\left(\left|f_{n x}-f_{n}(x)\right| \chi_{B E(\varepsilon, f)}-M\left(\left|f_{n x}-f_{n}(x)\right| b_{n}\right) \mid\right. \\
& \leqq \bar{M}_{x}|M| f_{x}-f(x)-f_{n x}+f_{n}(x)||+\bar{M}_{x}\left|M\left[\left|f_{n x}-f_{n}(x)\right|\left(\chi_{B E(\varepsilon, f)}-b_{n}\right)\right]\right| \\
& \leqq \bar{M}_{x}\left[M\left|f_{x}-f_{n x}\right|+M\left|f(x)-f_{n}(x)\right|\right]+2\left\|f_{n}\right\|_{\infty}\left\|\chi_{B E(\varepsilon, f)}-b_{n}\right\| \\
& \leqq 2\left\|f-f_{n}\right\|+2 / n \rightarrow 0 \quad \text { as } n \rightarrow \infty, \quad \text { by }(2) .
\end{aligned}
$$

Thus, as a function of $x$,

$$
M_{w}\left[|f(w+x)-f(x)| \chi_{B E(\varepsilon, f)}(w)\right] \in\{\mathrm{B}-\mathrm{AP}\}
$$


and (3) holds. We now show that

$$
\begin{aligned}
\bar{M}_{w} \bar{M}_{x}\left[|f(w+x)-f(x)| \chi_{B E(\varepsilon, f)}(w)\right] & \\
& =\lim _{n \rightarrow \infty} M_{w} M_{x}\left[\left|f_{n}(w+x)-f_{n}(x)\right| b_{n}(w)\right] .
\end{aligned}
$$

For fixed $w \in \mathbf{R}$,

$$
|f(w+x)-f(x)| \chi_{B E(\varepsilon, f)}(w) \in\{\mathrm{B}-\mathrm{AP}\},
$$

so $M_{x}\left[|f(w+x)-f(x)| \chi_{B E(e, f)}(w)\right]$ exists for each $w \in \mathrm{R}$. Arguing as before,

$$
\begin{aligned}
& \bar{M}_{w}\left|M\left(\left|f_{w}-f\right| \chi_{B E(\varepsilon, f)}(w)\right)-M\left(\left|f_{n w}-f_{n}\right| b_{n}(w)\right)\right| \\
& \leqq \bar{M}_{w}\left|M\left[\left(\left|f_{w}-f\right|-\left|f_{n w}-f_{n}\right|\right) \chi_{B E(\varepsilon, f)}(w)\right]\right| \\
& \quad \bar{M}_{w}\left|M\left(\left|f_{n w}-f_{n}\right| \chi_{B E(\varepsilon, f)}(w)\right)-M\left(\left|f_{n w}-f_{n}\right| b_{n}(w)\right)\right| \\
& \leqq \bar{M}_{w}|M| f_{w}-f-f_{n w}+f_{n}||+\bar{M}_{w}\left|M\left[\left|f_{n w}-f_{n}\right|\left(\chi_{B E(\varepsilon, f)}(w)-b_{n}(w)\right)\right]\right| \\
& \leqq \bar{M}_{w}|M| f_{w}-f_{n w}|+M| f-f_{n}\|+2\| f_{n}\left\|_{\infty}\right\| \chi_{B E(\varepsilon, f)}-b_{n} \| \\
& \leqq 2\left\|f-f_{n}\right\|+2 / n \rightarrow 0 \quad \text { as } n \rightarrow \infty, \text { by }(2) .
\end{aligned}
$$

Thus, as a function of $w$,

$$
M_{x}\left[|f(w+x)-f(x)| \chi_{B E(\varepsilon, f)}(w)\right] \in\{\mathrm{B}-\mathrm{AP}\}
$$

and (4) holds. As $f_{n}, b_{n} \in \alpha(\mathrm{R})$,

$$
M_{x} M_{w}\left[\left|f_{n}(w+x)-f_{n}(x)\right| b_{n}(w)\right]=M_{w} M_{x}\left[\left|f_{n}(w+x)-f_{n}(x)\right| b_{n}(w)\right]
$$

for all $n=1,2, \ldots$ Applying this to (3) and (4) gives

$$
\begin{aligned}
\bar{M}_{x} \bar{M}_{w}\left[|f(w+x)-f(x)| \chi_{B E(\varepsilon, f)}(w)\right] & =\bar{M}_{w} \bar{M}_{x}\left[|f(w+x)-f(x)| \chi_{B E(\varepsilon, f)}(w)\right] \\
& =\bar{M}_{w}\left[\left\|f_{w}-f\right\| \chi_{B E(\varepsilon, f)}(w)\right] \\
& \leqq \varepsilon \bar{\mu}(B E(\varepsilon, f)) .
\end{aligned}
$$

This proves (1), from which the theorem follows.

2.9 Lemma. Let $f \in L_{1, \mathrm{loc}}(\mathrm{R})$ and suppose that for some $\varepsilon>0$

$$
\left.\bar{M}_{x} \bar{M}_{u}|f(w+x)-f(x)| \chi_{B E(\varepsilon, f)}(w)\right]<\infty .
$$

Suppose $0<L_{i} \uparrow \infty$ as $i \rightarrow \infty$ and let $U$ be an open set in $R$. Then there exists $u \in U$ and a subsequence $\left\{L_{i}^{\prime}\right\}$ of $\left\{L_{i}\right\}$ such that

exists and is finite.

$$
\lim _{i \rightarrow \infty} \frac{1}{2 L_{i}^{\prime}} \int_{-L_{i^{\prime}}}^{L_{i^{\prime}}}\left|f_{u}\right| \chi_{B E(\varepsilon, f)} d \mu
$$

Proof. We may as well assume $U$ is bounded. We show first that there exists $u \in U$ and a subsequence $\left\{L_{i}{ }^{\prime \prime}\right\}$ of $\left\{L_{i}\right\}$ such that 


$$
\lim _{i \rightarrow \infty} \frac{1}{2 L_{i}{ }^{\prime \prime}} \int_{-L_{i^{\prime \prime}}}^{L_{i^{\prime \prime}}}|f(u+w)-f(u)| \chi_{B E(\varepsilon, f)}(w) d \mu(w)
$$

exists and is finite. Otherwise

$$
\bar{M}_{w}\left[|f(u+w)-f(u)| \chi_{B E(\varepsilon, f)}(w)\right]=\infty
$$

for all $u \in U$. Take $T_{0}$ such that $U \subset\left(-T_{0}, T_{0}\right)$. Then for every $T \geqq T_{0}$,

$$
\infty=\frac{1}{T} \int_{U} \bar{M}\left[\left|f_{x}-f(x)\right| \chi_{B E(\varepsilon, f)}\right] d \mu(x) \leqq \frac{1}{T} \int_{-T}^{T} \bar{M}\left[\left|f_{x}-f(x)\right| \chi_{B E(\varepsilon, f)}\right] d \mu(x),
$$

contrary to (1).

From the fact that (2) exists and is finite it follows that there exists $N<\infty$ such that

$$
\begin{aligned}
0 \leqq & \frac{1}{2 L_{i}^{\prime \prime}} \int_{-L_{i^{\prime \prime}}}^{L_{i^{\prime \prime}}}\left|f_{u}\right| \chi_{B E(\varepsilon, f)} d \mu \\
\leqq & \frac{1}{2 L_{i}^{\prime \prime}} \int_{-L_{i^{\prime \prime}}}^{L_{i^{\prime \prime}}}|f(u+w)-f(u)| \chi_{B E(\varepsilon, f)}(w) d \mu(w)+ \\
& +\frac{1}{2 L_{i}^{\prime \prime}} \int_{-L_{i^{\prime \prime}}}^{L_{i^{\prime \prime}}}|f(u)| \chi_{B E(\varepsilon, f)}(w) d \mu(w) \\
< & N \quad \text { for all } i=1,2, \ldots .
\end{aligned}
$$

Thus we may take $\left\{L_{i}^{\prime}\right\}$ to be a suitable subsequence of $\left\{L_{i}^{\prime \prime}\right\}$, proving the lemma.

2.10 Lemma. If $h \in \alpha(\mathrm{R})$, then $\bar{\mu}(E(\varepsilon, h))>0$ for every $\varepsilon>0$.

Proof. Take $\varepsilon>0$. Take $\delta>0$ such that $(-\delta, \delta) \subset E\left(\frac{1}{2} \varepsilon, h\right)$. Take $L>0$ such that $E\left(\frac{1}{2} \varepsilon, h\right) \cap(k L,(k+1) L) \neq \varnothing$ for all $k=0, \pm 1, \ldots$ Then

$$
\begin{aligned}
\bar{\mu}(E(\varepsilon, h)) & \geqq \varlimsup_{n \rightarrow \infty} \frac{1}{2 n L} \sum_{k=-n}^{n-1} \int_{k L}^{(k+1) L} \chi_{E(\varepsilon, h)} d \mu \\
& \geqq \varlimsup_{n \rightarrow \infty} \frac{1}{2 n L}(2 n \delta)=\delta / L>0 .
\end{aligned}
$$

2.11 Theorem. Let $f \in L_{1, \text { loc }}(\mathrm{R})$ satisfy (A2) and (B). Then $f \in\{\mathrm{B}-\mathrm{AP}\}$.

Proof. Take arbitrary $\delta>0$. For $x \in \mathrm{R}$ define $h(x)=\left\|f_{x}-f\right\|$. By 2.3, $h \in \alpha(\mathrm{R})$ and $E(\varepsilon, h)=B E(\varepsilon, f)$ for all $\varepsilon>0$. By $(\mathrm{B})$ and 2.5 there exists $\varepsilon$ in $T(h)$ such that 
(1) $\varepsilon<\delta$ and $\bar{M}_{x} \bar{M}_{w}\left[|f(x+w)-f(x)| \chi_{B E(\varepsilon, f)}(w)\right] \leqq \varepsilon \bar{\mu}(B E(\varepsilon, f))$.

By 2.10 we have $\bar{\mu}(B E(\varepsilon, f))>0$ so we may define

$$
g=\chi_{B E(\varepsilon, f)} / \bar{\mu}(B E(\varepsilon, f)) .
$$

By 2.7, $g \in\{\mathrm{B}-\mathrm{AP}\}$. Hence

$$
\left\{\begin{array}{l}
M g \text { exists and } M g=1 ; \\
0 \leqq g(x) \leqq\|g\|_{\infty}<\infty \text { for all } x \in \mathrm{R} .
\end{array}\right.
$$

From (1), (2) we get that

$$
\bar{M}_{x} \bar{M}_{w}[|f(x+w)-f(x)| g(w)]<\delta .
$$

Take $j \in\{1,2, \ldots\}$. By (A4) there exists a finite set $F_{j} \subset \mathrm{R}$ such that for every $y \in \mathrm{R}$ there is some $v \in F_{j}$ with $y=w+v$ for some $w \in B E(1 / j, f)$. Hence

$$
\left\|f_{y}-f_{v}\right\|=\left\|f_{w+v}-f_{v}\right\|=\left\|f_{w}-f\right\|<1 / j .
$$

As $B E(1 / j, f)$ contains a neighborhood of 0 , there is a neighborhood $U_{v}$ of each $v \in F_{j}$ such that the following holds: if $y \in R$ then there is some $v \in F_{j}$ such that

$$
\left\|f_{y}-f_{u}\right\|<2 / j \text { for all } u \in U_{v} .
$$

Let $F=\bigcup_{j=1}^{\infty} F_{j}$. We shall apply lemma 2.9. By (1), the hypotheses of 2.9 are satisfied. The role of $U$ in the lemma is to be taken by the sets $U_{v}$, $v \in F$. We enumerate the sets $U_{v}$, say $U_{1}, U_{2}, \ldots$ Take any sequence $\left\{T_{i}\right\}$ such that $0<T_{i} \uparrow \infty$ as $i \rightarrow \infty$. By applying 2.9 inductively and using a diagonal argument we get a subsequence $\left\{L_{i}^{\prime}\right\}$ of $\left\{T_{i}\right\}$ and a set $\left\{x_{1}, x_{2}, \ldots\right\} \subset \mathrm{R}$ such that $x_{j} \in U_{j}, j=1,2, \ldots$, and

$$
\lim _{i \rightarrow \infty} \frac{1}{2 L_{i}^{\prime}} \int_{-L_{i^{\prime}}}^{L_{i^{\prime}}}\left|f_{x_{j}}\right| \chi_{B E(e, f)} d \mu
$$

exists and is finite for all $j=1,2, \ldots$, Let $D=\left\{x_{1}, x_{2}, \ldots\right\}$. Because of (5) and the way $D$ is formed, we have that for every $\eta>0$ and every $y \in R$ there exists $x \in D$ such that

$$
\left\|f_{y}-f_{x}\right\|<\eta \text {. }
$$

If $x \in D$, then (2), (3) and the above considerations show that

$$
\begin{aligned}
0 & \leqq \varlimsup_{i \rightarrow \infty}\left|\frac{1}{2 L_{i}^{\prime}} \int_{-L_{i^{\prime}}}^{L_{i^{\prime}}} f_{x} g d \mu\right| \\
& \leqq\|g\|_{\infty}\left[\varlimsup_{i \rightarrow \infty} \frac{1}{2 L_{i}^{\prime}} \int_{-L_{i^{\prime}}}^{L_{i^{\prime}}}\left|f_{x}\right| \chi_{B E(\varepsilon, f)} d \mu\right]<\infty .
\end{aligned}
$$


As $D$ is countable, we may use a second diagonal argument to get a subsequence $\left\{L_{i}\right\}$ of $\left\{L_{i}^{\prime}\right\}$ such that

$$
\lim _{i \rightarrow \infty} \frac{1}{2 L_{i}} \int_{-L_{i}}^{L_{i}} f_{x} g d \mu
$$

is finite for all $x \in D$.

We now show that (7) exists and is finite for all $x \in \mathrm{R}$. Indeed take any $y \in \mathrm{R}$ and any $\eta>0$. Take $x \in D$ such that (6) holds. There exists $i_{0}$ such that

$$
\frac{1}{2 L_{i}} \int_{-L_{i}}^{L_{i}}\left|f_{y}-f_{x}\right| d \mu<\eta
$$

for all $i \geqq i_{0}$. Thus, for $i \geqq i_{0}$

$$
\left|\frac{1}{2 L_{i}} \int_{-L_{i}}^{L_{i}} f_{y} g d \mu-\frac{1}{2 L_{i}} \int_{-L_{i}}^{L_{i}} f_{x} g d \mu\right| \leqq\|g\|_{\infty} \frac{1}{2 L_{i}} \int_{-L_{i}}^{L_{i}}\left|f_{y}-f_{x}\right| d \mu<\eta\|g\|_{\infty} .
$$

Consequently, for large values of $i$,

$$
\frac{1}{2 L_{i}} \int_{-L_{i}}^{L_{i}} f_{y} g d \mu
$$

is within $2 \eta\|g\|_{\infty}$ of the finite number

$$
\lim _{i \rightarrow \infty} \frac{1}{2 L_{i}} \int_{-L_{i}}^{L_{i}} f_{x} g d \mu
$$

As $\eta>0$ is arbitrary, we get by the Cauchy criterion that

$$
\varphi(y)=\lim _{i \rightarrow \infty} \frac{1}{2 L_{i}} \int_{-L_{i}}^{L_{i}} f_{y} g d \mu
$$

is a finite number for all $y \in \mathrm{R}$. Now for $x, u \in \mathrm{R}$ we have

$$
|\varphi(u+x)-\varphi(x)| \leqq\|g\|_{\infty}\left\|f_{u+x}-f_{x}\right\| \leqq\|g\|_{\infty}\left\|f_{u}-f\right\| .
$$

Thus for every $\eta>0$,

$$
B E\left(\eta /\|g\|_{\infty}, f\right) \subset E(\eta, \varphi) .
$$

Using (A2), it follows that $\varphi \in \alpha(\mathrm{R})$.

We now show that $\|\varphi-f\|<\delta$. As $M[g]=1$, we get for any $x \in \mathrm{R}$ that 
Hence,

$$
\begin{aligned}
|\varphi(x)-f(x)| & =\lim _{i \rightarrow \infty}\left|\frac{1}{2 L_{i}} \int_{-L_{i}}^{L_{i}}[f(x+w)-f(x)] g(w) d \mu(w)\right| \\
& \leqq \bar{M}_{w}[|f(x+w)-f(x)| g(w)] .
\end{aligned}
$$

$$
\|\varphi-f\|=\bar{M}_{x}|\varphi(x)-f(x)| \leqq \bar{M}_{x} \bar{M}_{w}[|f(x+w)-f(x)| g(w)]<\delta,
$$

by (4). As $\delta>0$ is arbitrary, and $\varphi \in \alpha(R)$, the theorem follows.

MaIn Theorem. Let $f \in L_{1, \text { loc }}(\mathrm{R})$. Then $f \in\{\mathrm{B}-\mathrm{AP}\}$ if and only if $f$ satisfies (A1) and (B). The condition (A1) may be replaced by any of the equivalent conditions (A2), (A3) or (A4).

Proof. This follows from 2.1, 2.2, 2.8 and 2.11.

\section{Further comments.}

The most natural ways of weakening (A3) and (B) fail to give a characterization of $\{\mathrm{B}-\mathrm{AP}\}$. For example, (A3) alone will not characterize $\{\mathrm{B}-\mathrm{AP}\}$, as is illustrated by the example on page 5 of [5].

By the phrase " $B E(\varepsilon, f)$ has width" we mean that it is either of positive measure or of second category. We have seen that if $B E(\varepsilon, f)$ has width for every $\varepsilon>0$, then each $B E(\varepsilon, f)$ is in fact open. Condition (B) plus the requirement that each $B E(\varepsilon, f)$ have width fails to characterize $\{\mathrm{B}-\mathrm{AP}\}$, as is illustrated by the function $f(x)=x$ : it is certainly not $\mathrm{B}-\mathrm{AP}$, while $B E(\varepsilon, f)=(-\varepsilon, \varepsilon)$ and

for all $\varepsilon>0$.

$$
\bar{M}_{x} \bar{M}_{w}\left[|f(x+w)-f(x)| \chi_{B E(\varepsilon, f)}(w)\right]=0
$$

If for some $\varepsilon>0$, the set $B E(\varepsilon, f)$ has no width, then, by the main theorem, $f \notin\{\mathrm{B}-\mathrm{AP}\}$. It is conceivable, however, that if $f$ satisfies (B) and each $B E(\varepsilon, f)$ is relatively dense, then each $B E(\varepsilon, f)$ has width, whence $f \in\{\mathrm{B}-\mathrm{AP}\}$. In other words it is conceivable that the width requirement can be removed from (A3). We show that this is false by giving a function $f$ such that for every $\varepsilon>0$ the set $B E(\varepsilon, f)$ is relatively dense but has no width. (Hence $\mu(B E(\varepsilon, f))=0$ so that $f$ satisfies (B) trivially.) The function $f$ will also satisfy

$$
\lim _{T \rightarrow \infty} \frac{1}{2 T} \int_{-T}^{T} f d \mu \quad \text { exists . }
$$

We define $f$ as follows: On $[0,3)$ and on all intervals of the form $[3 n, 3 n+1), n \in\{ \pm 1, \pm 2, \ldots\}$, we define $f \equiv 0$. Suppose $k=3 n+1$ for some $n \in\{ \pm 1, \pm 2, \ldots\}$. Then on $[k, k+1)$ we define $f$ to be 


$$
\begin{aligned}
& (2 / 1) \chi_{(k, k+1 / 2)}+\left(2^{2} / 2\right) \chi_{(k+1 / 2, k+1 / 2+1 / 4)}+\ldots+ \\
& +\left(2^{n} /|n|\right) \chi_{(k+1 / 2+\ldots+1 / 2|n|-1, k+1 / 2+\ldots+1 / 2|n|)} .
\end{aligned}
$$

If $k=3 n+2$ for some $n \in\{ \pm 1, \pm 2, \ldots\}$, we define $f$ on $[k, k+1)$ to be the negative of $(2)$.

Let $S(n)=1+1 / 2+\ldots+1 / n$ and for $T>0$ let $n(T)$ be the smallest positive integer such that $T \leqq 3 n(T)$. For $T>0$ we have

$$
\begin{aligned}
\left|\frac{1}{2 T} \int_{-T}^{T} f d \mu\right| & \leqq \frac{1}{2 T}[2 S(n(T))] \\
& \leqq\left(\frac{n(T)}{3 n(T)-3}\right)\left(\frac{S(n(T))}{n(T)}\right) \\
& \leqq \frac{\log (n(T))+1}{n(T)} \rightarrow 0 \quad \text { as } T \rightarrow \infty .
\end{aligned}
$$

Thus $f$ satisfies (1).

Take any $i \in\{0, \pm 1, \ldots\}$. Due to the cancellations which occur when we subtract the graph of $f$ from the graph of $f_{3 i}$, we get that for $T>0$

$$
\begin{aligned}
& \frac{1}{2 T} \int_{-T}^{T}|f(x+3 i)-f(x)| d \mu(x) \\
& \quad \leqq(1 / 2 T)\left[k_{i}+4|i| S(|i|+n(T))\right] \\
& \quad \leqq\left(k_{i} / 2 T\right)+4|i|\left(\frac{|i|+n(T)}{6 n(T)-6}\right) \frac{S(|i|+n(T))}{(|i|+n(T))} \rightarrow 0 \text { as } T \rightarrow \infty ;
\end{aligned}
$$

here $k_{i}$ is a constant which covers the behavior of the graph of $f_{3 i}-f$ near zero. It follows that $\{0, \pm 3, \pm 6, \ldots\} \subset B E(\varepsilon, f)$ for every $\varepsilon>0$, whence each $B E(\varepsilon, f)$ is relatively dense.

Take $\varepsilon>0$. We shall show that $B E(\varepsilon, f)$ does not contain a neighborhood of 0 . Otherwise, there exists $0<\delta<1$ such that

$$
\left\|f_{\delta}-f\right\|<\varepsilon .
$$

For positive integers $n, k$ with $n>k$, let $S(k, n)=1 / k+\ldots+1 / n$. If $k>1$ is such that

$$
1 / 2+1 / 4+\ldots+1 / 2^{k-1}>\delta
$$

and $n>k$, then 


$$
\begin{aligned}
\frac{1}{6 n} \int_{-3 n}^{3 n}\left|f_{\delta}-f\right| d \mu & \geqq \frac{1}{6 n} \sum_{i=k+1}^{n} S(k, i) \\
& \geqq \frac{1}{6 n} \sum_{i=k+1}^{n}[\log i-\log k] \\
& \geqq \frac{1}{6 n}\left[\int_{k}^{n}(\log x) d \mu(x)-(n-k-1) \log k\right] \\
& =\frac{1}{6}[\log n-1-\log k+(k+\log k) / n] \rightarrow \infty \text { as } n \rightarrow \infty .
\end{aligned}
$$

This contradicts (3). Hence $B E(\varepsilon, f)$ does not contain a neighborhood of 0 . As $\varepsilon>0$ is arbitrary, none of the sets $B E(\varepsilon, f)$ have width, as was to be shown.

We do not know if (B) can be replaced by the stronger condition $(B)^{\prime}$ for every $\varepsilon>0$

$$
\bar{M}_{x} \bar{M}_{w}\left[|f(x+w)-f(x)| \chi_{B E(\varepsilon, f)}(w)\right] \leqq \varepsilon \bar{\mu}(B E(\varepsilon, f)) .
$$

Nor do we know if $\left\{\mathrm{B}^{p}-\mathrm{AP}\right\}$ can be characterized by inserting the parameter $p$ into $(\mathrm{A} i)$ and $(\mathrm{B}), 1 \leqq i \leqq 4,1<p<\infty$.

The authors wish to thank the referee for pointing out a serious error in the original manuscript.

\section{REFERENCES}

1. A. S. Besicovitch, Analysis of conditions of almost periodicity, Acta Math. 58 (1932), 217-230.

2. A. S. Besicovitch and $\mathrm{H}$. Bohr, Almost periodicity and generalized trigonometric series, Acta Math. 57 (1931), 203-292.

3. R. Doss, On generalized almost periodic functions, Ann. of Math. 59 (1954), 477-489.

4. R. Doss, On generalized almost periodic functions II, J. London Math. Soc. 37 (1962), 133-140.

5. E. Følner, On the structure of generalized almost periodic functions, Danske Vid. Selsk. Math. Fys. Medd. 21 No. 11 (1945), 1-30.

6. E. Følner, On the dual spaces of Besicovitch almost periodic spaces, Danske Vid. Selsk. Mat. Fys. Medd. 29 No. 1, (1954), 1-27.

7. P. R. Halmos, Measure theory, D. van Nostrand, Princeton - London - New York • Toronto, 1950.

8. E. Hewitt and K. A. Ross, Abstract Harmonic Analysis I (Grundlehren Math. Wiss. 115), Springer-Verlag, Berlin · Göttingen - Heidelberg, 1963.

9. J. L. Kelley, General Topology, D. van Nostrand, Princeton - London - New York · Toronto, 1955.

10. A. S. Kovanko, On a certain property and a new definition of generalized almost periodic functions of A.S. Besicovitch, Ukrain. Mat. Ž. 8 (1956), 273-288 (Russian). Amer. Math. Soc. Transl. (2) 25, (1963), 131-149. 